\title{
Ein Blick in die Schulpraxis: Diskussionspunkt Mathematikausbildung
}

Im Artikel „Ist Die Kompetenzorientierung wirklich Schuld? Ein Blick in die Schulpraxis" von Brigitte LutzWestphal äußern sich Lehrerinnen und Lehrer zur Kompetenzorientierung in der Schulpraxis. Diese Äußerungen sind ausschließlich positiv: Kompetenzorientierung sei „ein Gewinn“, „der einzig richtige Weg im Unterricht“, "gewinnbringend“, „[bedeutet] eine Verbesserung des Mathematikunterrichts". Sie unterscheiden sich damit deutlich von den Stellungnahmen, die ich im Jahr $2015 \mathrm{zu}$ diesem Thema von Lehrerinnen und Lehrern erhob.

Da ich im Moment unter Anderem im Rahmen des Qualitätspakts Lehre an den Verbesserungen der Studienbedingungen arbeite und so auf das Thema gestoßen wurde, hatte ich damals einen Vortrag hier an der Universität Bielefeld zur Zusammenfassung des damaligen Standes der Kompetenzdebatte in der Mathematik gehalten. Da$\mathrm{zu}$ hatte ich allen Lehrerinnen und Lehrern in meinem weiteren Bekanntenkreis die Frage gestellt, was sie von Kompetenzorientierung im Unterricht halten. Es wurden etwa 15 Lehrerinnen und Lehrer aller Fächer (Mathematik, Biologie, Wirtschaft, Physik, Deutsch u.a.) mit jahrzehntelanger Erfahrung an allen möglichen Schulformen (hauptsächlich Gymnasium, Gesamtschule, Berufskolleg), darunter zwei Schulleiter, mündlich befragt. Die Aussagen habe ich schriftlich festgehalten, meist in zusammengefasster Form. Die aussagekräftigsten habe ich wörtlich festgehalten. Diese Aussagen unterscheiden sich teils deutlich von denen im oben genannten Artikel. Da ich es für gewinnbringend halte diese Diskussion aufrichtig zu führen, finde ich es sinnvoll, ein möglichst umfassendes und komplettes Meinungsbild zu zeichnen. Um dazu beizutragen möchte ich diese Aussagen hier zusammenfassend wiedergeben.

Es ergaben sich zwei etwa gleich große Gruppen von Lehrerinnen und Lehrern. Für die eine Gruppe ist die Kompetenzorientierung eine Vorgabe von oben, die nicht in Frage gestellt wird. Es gibt Regeln, denen folgt man, die hinterfragt man nicht. Daher hatte diese Gruppe wenig zu dem Thema zu sagen. Bei der anderen Gruppe hat die Erwähnung von „Kompetenzorientierung“ Schleusen geöffnet. Zusammengefasst ist für diese Lehrerinnen

Mathematiklehrer an einer Gesamtschule in Rheinland-Pfalz: Mittelstufe (Sekundarstufe I)

Ich habe Glück mit meiner 7. Klasse. Eine quirlige, lebhafte und nette Gruppe, keine Kinder mit sonderpädagogischem Förderbedarf (Lern-, sozial-emotionale oder geistige Beeinträchtigung) und keine Flüchtlingskinder. und Lehrer Kompetenzorientierung eine aufoktroyierte Modeerscheinung, die sich gut anhört, die praxisfremd ist und guten Unterricht unnötig behindert. Einige OTöne aus der letzteren Gruppe: „So was [eine neue Mode] passiert alle 10 Jahre“ (drei Nennungen!), „Wir Lehrer werden bei so was nicht gefragt“, „Inhaltlich und methodisch habe ich meinen Unterricht nicht geändert, obwohl es jetzt Kompetenz heißt“, „Die Mehrheit macht da eher nicht mit“, „Früher hieß das ,Anforderungssituation“, heute heißt das ,Handlungssituation'. Da müssen dann viele Formulierungen geändert werden“, „Man macht nichts anders, es heißt nur anders", "Gleiche Sache, nur andere Worte". Dabei werden diese Vorgaben nicht als falsch empfunden: „Ich lese das, und denke: ja, genau das sollen meine Schüler können", aber als rigide und einengend, siehe oben, oder: „Wir müssen heute in den Klausuraufgaben z. B. alle zehn ,Zielformulierungen' einbauen. Das geht gar nicht." Eine positive Wirkung wurde den wechselnden Moden übrigens auch zugesprochen: da die Vorgaben regelmäßig wechseln, sind auch weniger engagierte Lehrerinnen und Lehrer gezwungen, ihr Lehrmaterial (Arbeitsblätter usw.) regelmäßig zu aktualisieren. Daneben herrscht übrigens unter den von mir befragten Lehrerinnen und Lehrern ein breiter Konsens, dass die Lehrpläne ausgedünnt wurden: „Der Lehrplan ist dünner geworden“, „Mittelstufenstoff ist ausgedünnt“, „Da wird vieles oberflächlicher gemacht als früher."

Mein Fazit aus obiger Erhebung war und ist, dass die Debatte um Kompetenzorientierung am Problem vorbeigeht, da das in der Schulpraxis eine untergeordnete Rolle spielt und in zehn Jahren eh niemand mehr von Kompetenz reden wird. Die eigentliche Frage muss lauten, wie zumindest zukünftigen MINT-Studierenden ermöglicht werden kann, an der Schule genug Mathematik zu lernen, um sie auf ein entsprechendes Studium genügend gut vorzubereiten.

\footnotetext{
Dr. Dirk Frettlöh, Universität Bielefeld, Technische Fakultät, AG Machine Learning, Universitätsstraße 25, 33615 Bielefeld frettloe@math.uni-bielefeld.de
}

In meinem Unterricht sitzen sehr schwache bis halbwegs starke Schüler. Einige werden die Schule mit der Berufsreife verlassen, viele die Allgemeine Hochschulreife anstreben. Die wirklich guten kommen erst gar nicht zu uns an die Schule oder sie wechseln bald ans Gymnasium. 
In einem Teil der Unterrichtsstunden habe ich nur jeweils die Hälfte der Klasse, die andere Hälfte hat in dieser Zeit Englisch, dann wird gewechselt. In diesen Stunden bleibt etwas mehr Zeit für einzelne Schüler, aber auch hier sind wieder alle Leistungsniveaus vertreten und am Ende muss ich in beiden Gruppen gleich weit sein, denn in der nächste Stunde ist die Klasse wieder vollständig.

Die ganz schwachen Schüler müsste ich an die Hand nehmen, mich neben sie setzen, individuell mit ihnen arbeiten, so dass sie eine Chance haben mitzukommen. Aber was machen dann die anderen? Die beschäftigen sich nicht einfach sinnvoll mit Arbeitsblättern. Das funktioniert in der Theorie, aber die Schüler, die das könnten und machen würden, gibt es bei mir nicht.

Viele neue Methoden habe ich ausprobiert, aber bald gemerkt, dass davon in der Regel nur starke Schüler profitieren. Die schwachen brauchen eine enge Führung, eine direkte Anleitung und konkrete Rückmeldung. Das kann kein Arbeitsblatt und auch kein Mitschüler leisten. Also halte ich überwiegend lehrerzentrierten Unterricht (das hieß früher: Frontalunterricht).

Hausaufgaben und Materialien mitzubringen, das scheint heute optional zu sein. Im Mittel haben bei mir etwa $25 \%$ der Schüler keine oder nur teilweise ihre Materialien dabei bzw. die Hausaufgaben gemacht. Im Moment haben wir noch einen Lehrplan, der sinnvollerweise zwischen den existierenden Schulabschlüssen differenziert. Darin wird darauf Wert gelegt, dass für Schüler mit dem Ziel Berufsreife (Hauptschulabschluss) mehr Zeit für das Wiederholen von praxisrelevanten Inhalten besteht. (Zinsrechnung, Flächenberechnung, ...) Wie wiederhole ich diese Inhalte aber mit den betreffenden Schülern, wenn ich gleichzeitig mit den anderen in der Klasse das Rüstzeug für die Oberstufe erarbeiten soll?

Oberstufe (Sekundarstufe II)

In der Oberstufe ist alles besser, nahm ich an. Die Schüler streben die Allgemeine Hochschulreife an und sind frei-

Unter der Überschrift „Mathematische Lernvoraussetzungen für MINT-Studiengänge aus Sicht der Hochschulen“ wurden in den Mitteilungen 25-4 (2017) Ergebnisse des MaLeMINT-Projekts vorgestellt. Auf Seite 242, rechte Kolumne, Mitte findet man: „Die Ergebnisse zeigen, dass Seitens der Hochschullehrenden nicht nur Kenntnisse mathematischer Inhalte und Arbeitsweisen erwartet werden, sondern darüber hinaus auch ein Verständnis der Mathematik als wissenschaftliche Disziplin [...].“ Damit schließen sich die Hochschullehrenden mehrheitlich offenbar dem Postulat von Lisa Hefendehl-Hebeker an, wonach Mathematikunterricht erlebbar machen solle, wie mathematische Wissensbildung geschieht.

Mit großer Verwunderung muss man wenige Zeilen später lesen: „Zur Notwendigkeit der Lernvoraussetzung ,Entwickeln und Formulieren mathematischer Beweise zu einer gegebenen Behauptung' konnte [...] kein Konsens festgestellt werden." Demnach soll nach dem Wunsch willig in der Schule. Die Realität sieht anders aus. Termumformungen, quadratische Gleichungen und Potenzrechengesetze, all das sollte aus der Mittelstufe bekannt und verfügbar sein, aber oft sind es nur rudimentäre Kenntnisse und Fertigkeiten. Am Anfang der 11. Klasse muss ich solche Rechentechniken mühsam mit den Schülern einüben, damit der eigentliche Fachunterricht der Oberstufe überhaupt beginnen kann. Diese Zeit fehlt natürlich im weiteren Verlauf und wir verbleiben auf einem sehr einfachen Niveau des Einübens von Rechenroutinen, um später die Aufgaben im schriftlichen Abitur lösen zu können. Guter Mathematikunterricht sieht für mich anders aus. Nachdenken über Mathematik findet im Unterricht nicht statt, solange die Grundlagen so dürftig sind.

In Rheinland-Pfalz gibt es noch 5-stündige Leistungskurse im Gegensatz zu 3-stündigen Grundkursen. 24 Schüler haben in meinem Leistungskurs in der 11. Klasse angefangen, zu Beginn der 12. Klasse war nur noch die Hälfte da. Die anderen haben die Schule verlassen oder wiederholen die Klasse, manche auch wieder mit Mathematik als Leistungskurs.

Im Leistungskurs treffe ich immerhin auf eine mäßige Leistungsbereitschaft, wenn auch die Leistungsfähigkeit beschränkt ist. Dagegen fehlt im Grundkurs oft auch noch die Bereitschaft, fehlende Grundlagen nachzuarbeiten. Rechnungen scheitern regelmäßig an elementaren algebraischen Umformungen oder der Bruchrechnung. Bedenklich finde ich, dass Lehrkräfte ohne Lehrbefähigung für die Sekundarstufe II in den Grundkursen unterrichten. Die Leistungskurse sind Ihnen (noch) verwehrt.

So kann eine verantwortliche mathematische Schulbildung nicht funktionieren.

$$
\begin{gathered}
\text { Oliver Großhans, Im Goldschmied 12,67152 Ruppertsberg } \\
\text { ol.grosshans@googlemail.com }
\end{gathered}
$$

vieler Hochschullehrer/innen in den Köpfen von Schüler/innen ein Verständnis für Mathematik als wissenschaftliche Disziplin entfaltet werden, ohne das in diesen Köpfen je ein mathematischer Beweis zu einer gegebenen Behauptung entwickelt wurde.

Damit liegt eine große Gruppe der Hochschullehrenden ganz auf der Linie des aktuellen mathematikdidaktischen Trends. Im Rahmen dieses Trends wird sogar vereinzelt bezweifelt, dass es ein Verständnis der Mathematik als wissenschaftliche Disziplin überhaupt geben kann. Denn dazu müsste man der Mathematik typische Wesenszüge zugestehen, die überhaupt erst einmal beschrieben werden müssten. Von einer solchen Beschreibung ist aber die aktuelle Mathematikdidaktik und insbesondere die Liste der Kompetenzen K1 bis K6 weiter entfernt, denn je.

Roland Schröder, Dehningstraße 26, 29223 Celle florola@gmx.de 


\title{
Vorstellung der Kandidaten für die Wahl zum DMV-Präsidium
}

\author{
Der Präsident der DMV ruft alle DMV-Mitglieder auf, sich an der Wahl von Präsidiumsmitgliedern \\ für die Amtszeit ab dem 1.1.2019 zu beteiligen. \\ Bei der Wahl sind drei Präsidiumsplätze zu besetzen: Ein Platz im Vorstand (Schriftführer) und zwei \\ reguläre Präsidiumsplätze. Es bewerben sich drei Kandidaten. Diese stellen sich hier vor. \\ Die Wahlunterlagen liegen diesem Heft bei.
}

Prof. Dr. Daniel Grieser

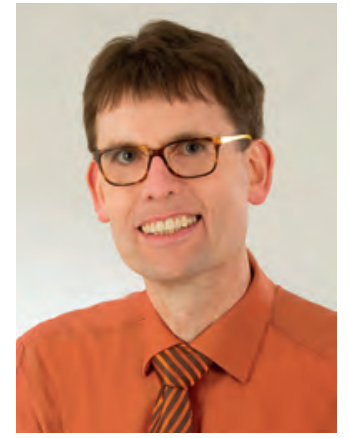

Geboren 1964 in Berlin, Studium der Mathematik und Physik an der Freien Universität Berlin, 1988 Diplom mit Schwerpunkt Kombinatorik, 1992 Promotion an der University of California, Los Angeles im Bereich Spektraltheorie partieller Differentialoperatoren, 1992-95 C.L.E. Moore Instructor am MIT (Massachusetts Institute of Technology), 2002 Habilitation an der Humboldt-Universität Berlin, 2002-2005 als Heisenberg-Stipendiat am MIT und an der Universität Bonn, seit 2005 Professor an der Carl von Ossietzky-Universität Oldenburg, Institutsdirektor 20092011, Organisator zahlreicher öffentlicher Veranstaltungen sowie Schüleraktivitäten zur Mathematik, seit 2015 Präsidiums- und Vorstandsmitglied (als Schriftführer) der DMV.

Der Schwerpunkt meiner Forschung liegt in der Spektraltheorie partieller Differentialoperatoren mit Beziehungen zur mathematischen Physik, der mikrolokalen Analysis und der globalen Analysis. Dabei interessiert mich besonders die Weiterentwicklung systematischer Methoden zur Untersuchung singulärer Probleme, wie sie zum Beispiel bei natürlichen geometrischen Differentialoperatoren auf singulären und nicht-kompakten Räumen oder bei den geometrisch oder physikalisch motivierten Problemen des semiklassischen oder des adiabatischen Limes auftreten.

In der Lehre liegt mir besonders daran, dass Studierende möglichst früh einen entdeckenden und fragenden Zugang zur Mathematik bekommen und dadurch die wunderbaren Methoden und Theorien, welche die Mathematik bereitstellt, nicht nur kennen-, sondern auch schätzen lernen. Zu diesem Zweck und mit dem Ziel, den Übergang Schule - Hochschule verträglicher zu gestalten, habe ich das Modul Mathematisches Problemlösen und Beweisen entwickelt, das 2014 mit dem ars legendi Fakultätenpreis ausgezeichnet wurde. Autor der Bücher Mathematisches Problemlösen und Beweisen und Analysis $I$.

Ich sehe die Hauptaufgaben der DMV darin, dass sie die Mathematik gegenüber der Politik und in der Gesellschaft vertritt und sichtbar macht und dass sie die Vernetzung mathematisch Tätiger in verschiedenen Berufsfeldern (Industrie, Hochschule, Schule) befördert. Sie kann und sollte sich in wichtigen mathematikbezogenen Themen innerhalb und außerhalb der mathematischen Community positionieren und engagieren, z.B. in den aktuellen Diskussionen über Mathematik in der Schule oder zur Bibliometrie. In diesen und den daraus erwachsenden spezifischen Aufgaben möchte ich die DMV durch meine erneute Kandidatur als Schriftführer und Präsidiumsmitglied unterstützen.

\section{Matthias Lippert}

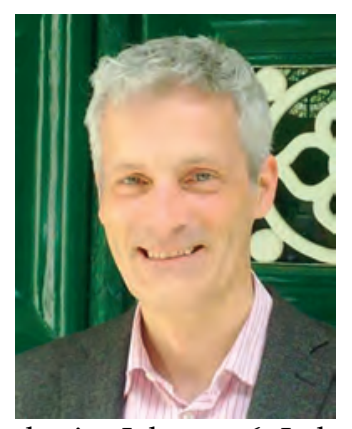

Geboren 1967; DiplomStudium in Mathematik an der Universität Hamburg, der Johann-Wolfgang-GoetheUniversität Frankfurt am Main und der Rheinischen FriedrichWilhelms-Universität in Bonn; Diplom in Mathematik (Themenbereich algebraische Zahlentheorie) bei Herrn Prof. Harder im Jahr 1996; Lehramtsstudium Physik an der Rheinischen Friedrich-Wilhelms-Universität in Bonn; erstes

Staatsexamen im Jahr 1997; zweites Staatsexamen im Jahr 2000; von 2000 bis 2011 Lehrer für Mathematik und Physik und seit 2007 stellvertretender Schulleiter am Gymnasium Schwertstraße in Solingen; seit 2011 Schulleiter am Städtischen Röntgen-Gymnasium in Remscheid.

Meine fachlichen Schwerpunkte liegen im Bereich der Unterrichtsentwicklung für den Mathematikunterricht der gymnasialen Oberstufe und in der Förderung von mathematisch interessierten Schülerinnen und Schülern im Rahmen von Schülerwettbewerben und MathematikAGs. In diesem Zusammenhang arbeite ich im Auftrag des nordrheinwestfälischen Schulministeriums in unter- 